\title{
POINTSAMPLER: A GIS TOOL FOR POINT INTERCEPT SAMPLING OF DIGITAL IMAGES
}

\author{
DAVID L. GOBBETT ${ }^{1}$ AND ANDRE ZERGER ${ }^{2}$ \\ ${ }^{I}$ CSIRO Sustainable Agriculture Flagship / CSIRO Ecosystem Sciences, PMB 2 Glen \\ Osmond, SA 5064, Australia; ${ }^{2}$ Environmental Information Services, Bureau of \\ Meteorology, GPO Box 2334, ACT 2600, Australia
}

\begin{abstract}
Close-range digital photography to assess vegetation cover is useful in disciplines ranging from ecological monitoring to agricultural research. An on-screen point intercept sampling method, which is analogous to the equivalent field based method, can be used to manually derive the percentage occurrence of multiple cover classes within an image. PointSampler is a GIS embedded tool that provides a semi-automated approach for performing point intercept sampling of digital images, and which integrates with existing GIS functionality and workflows. We describe and illustrate the two general applications of this tool, in efficiently deriving primary ecological data from digital photographs, and for the generation of validation data to complement automated image classification of a time series of groundcover images. The flexible design and GIS integration of PointSampler allows it to be put to a wide range of similar uses.
\end{abstract}

Key words.- Classification, groundcover, image assessment, photography, vegetation

Close range digital imagery is useful in research and monitoring where the proportions of different types of cover need to be assessed. Its applications include arid vegetation assessment (Laliberte et al. 2007), green vegetation cover (Liu et al. 2012), flower number estimation (Adamsen et al. 2000), crop canopy coverage (Purcell 2000), forest understory foliar coverage (Macfarlane and Ogden 2012) and lichen cover and biomass estimation (Bowker et al. 2008). The benefits of digital imagery over field based methods include reduced fieldwork time and data collection costs, which can enable data collection at a larger number of sites, plots or quadrats. Automated photography can also enable the capture of data at higher temporal frequency (e.g. hourly, daily). The rapid increase in a use of Unmanned Aerial Vehicles as platforms for image capture has expanded the use of digital imagery. A further benefit of digital images is that they can be retained for future reference or analysis for purposes other than they were originally collected.

Typically, close range images are used to determine attributes such as percentage vegetation cover within a number of classes, canopy cover or other presence-absence estimates. In ground cover vegetation analyses, such classes might include live vegetation, flowers, bare soil, rock or perhaps assign vegetation to particular types. The same techniques can be applied in a range of other research and monitoring domains. By classifying an image into discrete classes, the proportion of those classes can be used to assess change at a single point over time, or at points along a transect, or to facilitate comparisons between different sites.
Where large numbers of images are captured, either across a spatial extent, or at at intervals as a time-series, it may be appropriate to utilize automated image analysis methods such as supervised classification. Supervised classification assigns the pixels of an image to classes by determining the fit with previously input signatures, and generally treat each pixel as a discrete entity. Object oriented classification methods also incorporate analysis of shape and context in classifying an image. Regardless of the automated method used, a classification process needs to be validated. One approach is to undertake actual field observations, or ground truthing, but another option is to manually generate calibration or validation datasets from a subset of images to be statistically compared with the results of automated classification methods. Alternatively, manual image analysis may also be used as a primary data collection method.

The point intercept method (PIM; also known as point contact or point frequency method) is a wellestablished method for estimating vegetation cover proportions (e.g. Whitman and Siggeirsson 1954; Heady et al. 1959; Brun and Box 1963). In fieldwork the PIM involves counting the proportion of cover types under randomly or regularly placed pins. PIM is most suitable for single-layer vegetation, and has a lower level of subjectivity than visual estimation methods (Bråkenhielm and Qinghong 1995), although is less suitable for recording rare species (Moen et al. 2007). The method allows input from experts with specialized knowledge, or alternatively can be applied by an operator with a relatively low skill level, provided that appropriate classification 
rules are supplied. Applying a PIM to assessing cover in digital imagery is very similar to its use in a field situation.

Developing workflows that incorporate software tools and scripting can be essential for efficiently dealing with larger numbers of images captured in such studies. Automated image analysis methods can be used to determine percentage cover of different classes. Geographic information systems (GIS) include many features useful for the handling of imagery and combining them with related datasets. Scripting tools such as Python (Python Software Foundation 2011) provide the ability to organize and manipulate images and can provide the 'glue' to link various processing steps into a reusable workflow. While automated processes facilitate the processing of large number of images, it can be necessary to perform manual assessments of images, using methods such as the PIM, in order to validate automated processing. Manual image assessment can be time consuming, so there is a key niche for a software tool to provide a semi-automated approach to image assessment.
We evaluated two existing software tools for their suitability in assessing vegetation cover in digital imagery. VegMeasure (Johnson et al. 2003) is a semi-automated image classification tool that provides a variety of spectral band algorithms which can be calibrated by an operator using a point intercept method. VegMeasure is most suited to performing binary classifications, such as calculating cover of crop and bare ground, and did not readily accommodate our need to classify images into four or five classes. SamplePoint (Booth et al. 2006) is a tool for image classification using a point intercept method and supports a large number of cover classes. Cagney et al. (2011) estimated that on screen image sampling using SamplePoint took about one third of the time taken by a field based point intercept method. However, SamplePoint does not allow the re-use of the same set of randomly generated sample points to facilitate the comparison of changes in individual points over time. Further, because neither of these tools readily integrated with our existing GIS workflows, we set about developing our own tool which we named PointSampler.

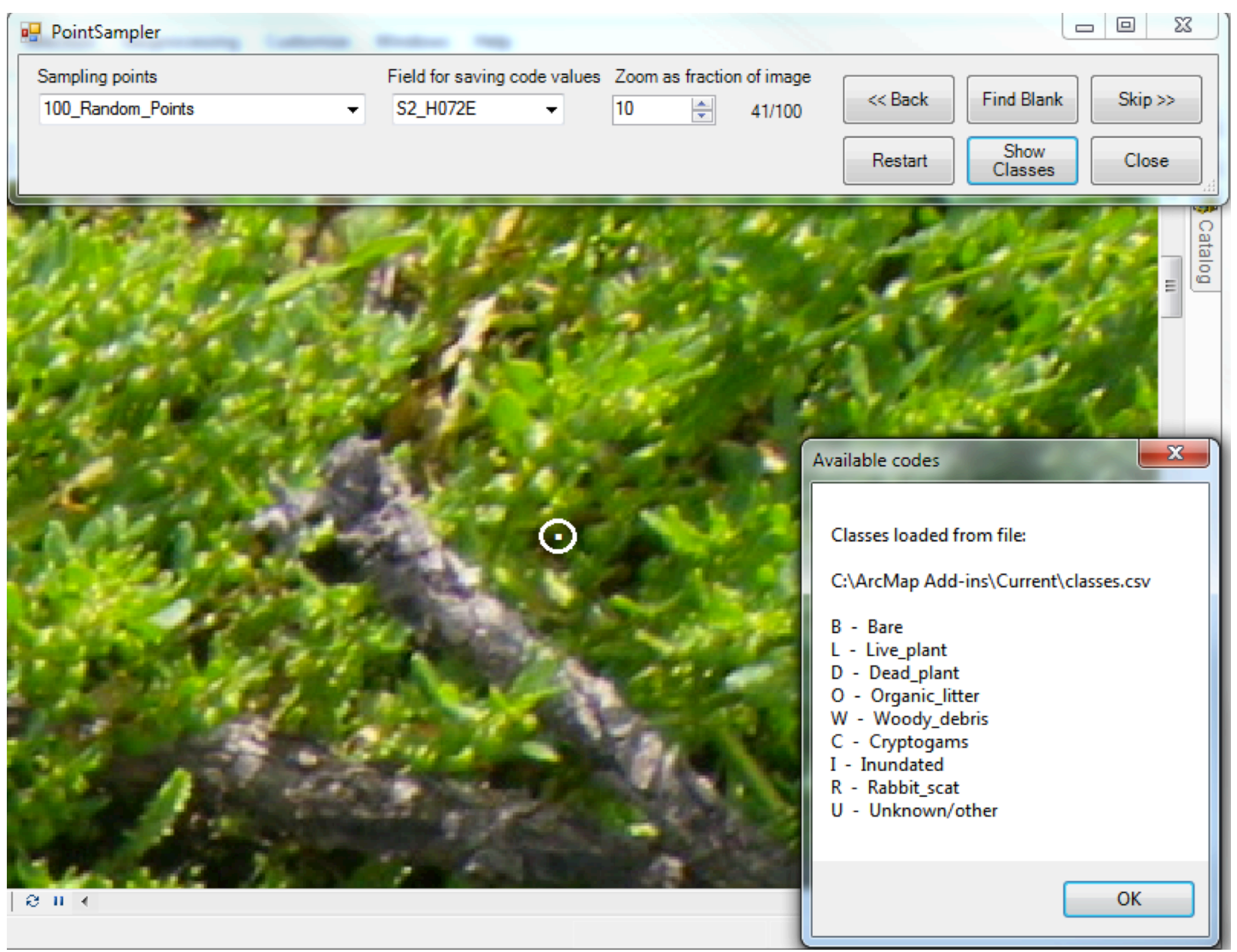

Figure 1. PointSampler being used to classify images within ArcGIS. 


\section{POINTSAMPLER DEVELOPMENT}

PointSampler was developed in Visual Basic .Net and is installed as an Add-in for ArcGIS version 10 (ESRI, Redmond, CA). PointSampler (Fig. 1) makes use of inbuilt ArcGIS functionality, such as the ability to load and display images and overlay Shapefiles, apply symbology, zoom and pan. Consequently, the user interface of PointSampler is kept simple, and is straightforward to use for anyone familiar with ArcGIS. Being embedded within ArcGIS also adds the ability to work with georeferenced images, work to fixed scale and potentially to calculate areas, overlay ancillary data or spatial datasets, and to compare multiple coincident images.

When launched, the operator assesses the cover class at a point displayed centrally on the screen then types the corresponding character code. The image then zooms automatically to the next point to be classified. The character codes are defined in a text file and can easily be modified to different needs.

For each set of images to be classified, a Shapefile contains the sample points and also stores the coded values for each point. The points to be sampled can be generated manually, or with tools such as Geospatial Modelling Environment (Beyer 2011) and may form a regular grid or random arrangement. For each image to be processed a data field is needed in the Shapefile to store the coded values. This field can be added manually or, for example, through an ancillary Python script which could create the Shapefile and add all necessary fields as part of the image processing workflow. After coding with PointSampler is completed, the Shapefile dataset can be used within ArcGIS or exported for further analysis.

\section{APPLICATIONS OF POINTSAMPLER}

There are many possible uses for PointSampler. Two key areas in which its value has been demonstrated are in the generation of primary dataset (Fig 2a), and to assist in the generation of a dataset used to validate an automated image classification process (Fig 2b). An example of each of these cases is presented here.

\section{Generation of a Primary Dataset}

PointSampler was as part of an assessment of floodplain woodland structure and condition (McGinness et al. 2013) to streamline the capture of groundcover data along transects within study sites. Photographs were taken $1.5 \mathrm{~m}$ above the ground using 12 megapixel compact digital cameras. Prior to analysis using PointSampler, the individual images were named so as to identify the site at which they were captured, but no other image preprocessing was performed. PointSampler was effectively used to capture a range of attributes from each image,

(a)

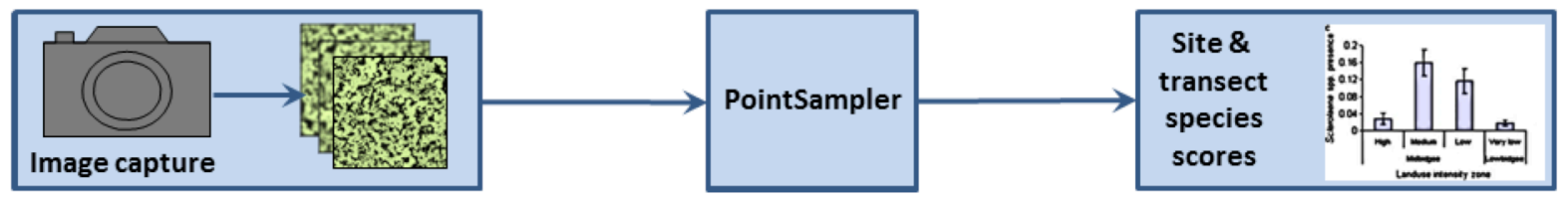

(b)

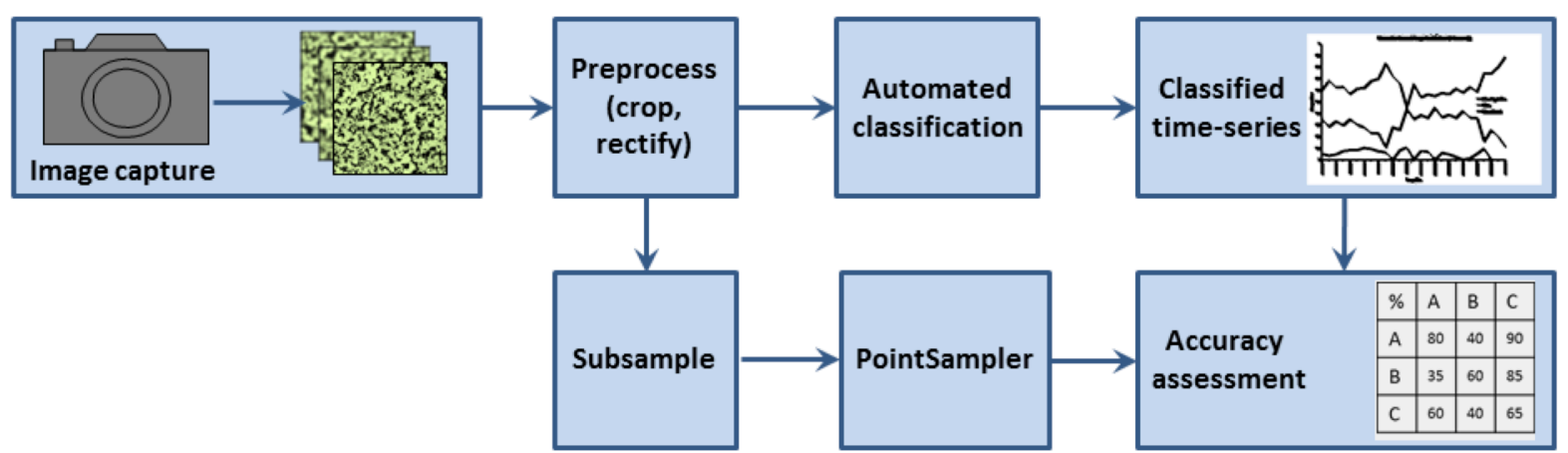

Figure 2: Applications of PointSampler in (a) primary data capture and (b) generation of a dataset for validating an automated image classification process. 
including the presence of three indicator plant species. The camera images did not need to be spatially referenced since there was no intention to compare repeat images of the same sites, and so they could be processed with PointSampler without additional processing. In this case, the use of PointSampler resulted in the efficient generation of primary data for each of the study sites.

\section{Generation of a Dataset for Validation of Automated Classifications}

In a ground-cover monitoring project, daily images of vegetation plots were captured over a six month period using fixed, downward pointing, consumer grade, weatherproof digital cameras (Zerger et al. 2012). To assess changes in groundcover we focused on five classes, live (L), attached litter (A), detached litter (D), bare ground (B) and other $(\mathrm{O})$. Automated processing was used to apply a Maximum Likelihood Classifier method within ENVI (ITT Visual Solutions, 2008) to detect trends in cover proportions over the entire time series. Image preparation included georeferencing and cropping each image to the plot boundary, which enabled appraisal of change at individual points in sequential images. In a similar way to Rotz et al. (2008) we required a validation dataset based on manual image assessment by a skilled operator. PointSampler was used to classify 100 random points from weekly images selected from the larger timeseries. In this case, PointSampler enabled the generation of a validation dataset used to assess the automated image classification process.

While performing the manual assessments using PointSampler, some subjectivity became apparent in the differentiation of classes which tend to occur across a gradient, such as from A to D and D to B. It was helpful to elucidate clear rules to assist the operator in performing the classifications. For a detailed description of these rules see Zerger et al. (2012). Overall PointSampler enabled the operator to process the images far more rapidly than would otherwise have been possible with a more manual process.

\section{DISCUSSION}

The above examples illustrate the utility of PointSampler for the collection of primary data for field research, and for generating a validation dataset used in an automated image classification process. In both case studies, clearly defined rules were needed to assist the operator in the classification task. In both cases PointSampler enabled the processing of larger numbers of images than would otherwise have been feasible in the available time.
Importantly, the integration of PointSampler within a GIS allows it to be incorporated into image processing workflows that can be automated with the Python scripting language. For example, using Python, cover class scores from a series of images using PointSampler could be summarized, or cross tabulated against reference images to calculate accuracy scores such as user's accuracy, producer's accuracy and kappa coefficients (Congalton 1991).

We intend to update PointSampler from time-totime to accommodate new versions of ArcGIS software. PointSampler benefits from tight integration within the ArcGIS user interface, which however precludes it from use with different GIS software. While PointSampler is a useful tool in its current form, a number of enhancements are being considered for a future version. These include the addition of program features to simplify editing of the classification codes, Shapefile management (such as simplifying the addition of a field for a new image), a training mode - similar to that offered in SamplePoint, which allows inexperienced users to compare their classification choices against those of an expert, and a function to generate producer's and user's accuracy statistics (Congalton 1991) against equivalent automatically classified images.

The use of low cost digital photography for primary field data collection and assessment, such as in estimating proportional composition of groundcover classes, can be substantially streamlined using PointSampler. Its use is particularly appropriate where GIS workflows form part of the image analysis process, and enables large numbers of images to be manually assessed efficiently. The versatility of PointSampler allows it to be applied to a range of similar uses, in any domain in which classification and sampling of digital images is used. As well as applications of the two types illustrated here, it is conceivable that PointSampler could be used for the generation of training datasets for input into automated processing.

The PointSampler ArcGIS Add-in can be downloaded from the CSIRO website ${ }^{1}$.

\section{ACKNOWLEDGEMENTS}

Feedback provided about PointSampler by various colleagues, especially Micah Davies and Heather McGinness, is appreciated. Two anonymous reviewers provided valuable comments and suggestions.

\footnotetext{
${ }^{1}$ http://www.csiro.au/PointSampler
} 


\section{REFERENCES}

Adamsen, F. J., T. A. Coffelt, J. M. Nelson, E. M. Barnes, and R. C. Rice. 2000. Method for using images from a color digital camera to estimate flower number. Crop Sci 40:704-709.

Beyer, H. L. 2011. Geospatial Modelling Environment (version 0.5.3 Beta)

Booth, D., S. Cox, and R. Berryman. 2006. Point sampling digital imagery with 'Samplepoint.' Environ Monit Assess 123:97-108.

Bowker, M. A., N. C. Johnson, J. Belnap, and G. W. Koch. 2008. Short-term monitoring of aridland lichen cover and biomass using photography and fatty acids. J Arid Environ 72:869-878.

Bråkenhielm, S. and L. Qinghong. 1995. Comparison of field methods in vegetation monitoring. Water Air Soil Poll 79:75-87.

Brun, J. M. and T. W. Box. 1963. A comparison of line intercepts and random point frames for sampling desert shrub vegetation. J Range Manage 16:21-25.

Cagney, J., S. E. Cox, and D. T. Booth. 2011. Comparison of point intercept and image analysis for monitoring rangeland transects. Rangeland Ecol Manag 64:309315.

Congalton, R. G. 1991. A review of assessing the accuracy of classifications of remotely sensed data. Remote Sens Environ 37:35-46.

Heady, H. F., R. P. Gibbens, and R. W. Powell. 1959. A Comparison of the charting, line intercept, and line point methods of sampling shrub types of vegetation. J Range Manage 12:180-188.

Johnson, D., M. Vulfson, M. Louhaichi, and N. Harris. 2003. Vegmeasure v1.6 user's manual. Department of Rangeland Resources, Oregon State University, Corvallis, Oregon.

Laliberte, A. S., A. Rango, J. E. Herrick, E. L. Fredrickson, and L. Burkett. 2007. An object-based image analysis approach for determining fractional cover of senescent and green vegetation with digital plot photography. J Arid Environ 69:1-14.

Liu, Y., X. Mu, H. Wang, and G. Yan. 2012. A novel method for extracting green fractional vegetation cover from digital images. J Veg Sci 23:406-418.

Macfarlane, C. and G. N. Ogden. 2012. Automated estimation of foliage cover in forest understorey from digital nadir images. Methods Ecol Evol 3:405-415.

McGinness, H. M., A. D. Arthur, M. Davies, and S. McIntyre. 2013. Floodplain woodland structure and condition: the relative influence of flood history and surrounding irrigation land use intensity in contrasting regions of a dryland river. Ecohydrology 6:201-213.

Moen, J., Ö. Danell, and R. Holt. 2007. Non-destructive estimation of lichen biomass. Rangifer 27:41-46.

Purcell, L. C. 2000. Soybean canopy coverage and light interception measurements using digital imagery. Crop Sci 40:834-837.

Python Software Foundation. 2011. Python programming language.

Rotz, J. D., A. O. Abaye, R. H. Wynne, E. B. Rayburn, G. Scaglia, and R. D. Phillips. 2008. Classification of digital photography for measuring productive ground cover. Rangeland Ecol Manag 61:245-248.

Whitman, W. C. and E. I. Siggeirsson. 1954. Comparison of line interception and point contact methods in the analysis of mixed grass range vegetation. Ecology 35:431-436.

Zerger, A., D. Gobbett, C. Crossman, P. Valencia, T. Wark, M. Davies, R. N. Handcock, and J. Stol. 2012. Temporal monitoring of groundcover change using digital cameras. Int J Appl Earth Obs Geoinformation 19:266-275. 